GA-A23960

\title{
ATOMIC PHYSICS PROCESSES IMPORTANT TO THE UNDERSTANDING OF THE SCRAPE-OFF LAYER OF TOKAMAKS
}

W.P. WEST, B. GOLDSMITH, T.E. EVANS, and R.J. OLSON 


\section{DISCLAIMER}

This report was prepared as an account of work sponsored by an agency of the United States Government. Neither the United States Government nor any agency thereof, nor any of their employees, makes any warranty, express or implied, or assumes any legal liability or responsibility for the accuracy, completeness, or usefulness of any information, apparatus, product, or process disclosed, or represents that its use would not infringe privately owned rights. Reference herein to any specific commercial product, process, or service by trade name, trademark, manufacturer, or otherwise, does not necessarily constitute or imply its endorsement, recommendation, or favoring by the United States Government or any agency thereof. The views and opinions of authors expressed herein do not necessarily state or reflect those of the United States Government or any agency thereof. 


\title{
ATOMIC PHYSICS PROCESSES IMPORTANT TO THE UNDERSTANDING OF THE SCRAPE-OFF LAYER OF TOKAMAKS
}

\author{
by \\ W.P. WEST, B. GOLDSMITH, ${ }^{*}$ T.E. EVANS, and R.J. OLSON†
}

This is a preprint of a paper to be presented at Third International Conference on Atomic and Molecular Data and Their Applications, Gatlinburg, Tennessee, April 24-26, 2002 and to be published in the Plroceedings.

*Student, University of California at San Diego, La Jolla, California. †Student, University of Missouri-Columbia, Columbia, Missouri.

\author{
Work supported by \\ the U.S. Department of Energy under \\ Contract No. DE-AC03-99ER54463
}




\title{
Atomic Physics Processes Important to the Understanding of the Scrape-Off Layer of Tokamaks
}

\author{
W.P. West, B. Goldsmith, ${ }^{(a}$ T.E. Evans, R.E. Olson ${ }^{(b}$ \\ General Atomics, P.O. Box 85608, San Diego, California 92186-5608 \\ ${ }^{(a}$ Student, University of California - San Diego, La Jolla, California 92093-0319 \\ ${ }^{(b}$ University of Missouri, Dept. of Physics, Rolla, Missouri 65401
}

\begin{abstract}
The region between the well-confined plasma and the vessel walls of a magnetic confinement fusion research device, the scrape-off layer (SOL), is typically rich in atomic and molecular physics processes. The most advanced magnetic confinement device, the magnetically diverted tokamak, uses a magnetic separatrix to isolate the confinement zone (closed flux surfaces) from the edge plasma (open field lines). Over most of their length the open field lines run parallel to the separatrix, forming a thin magnetic barrier with the nearby vessel walls. In a poloidally-localized region, the open field lines are directed away from the separatrix and into the divertor, a region spatially separated from the separatrix where intense plasma wall interaction can occur relatively safely. Recent data from several tokamaks indicate that particle transport across the field lines of the SOL can be somewhat faster than previously thought. In these cases, the rate at which particles reach the vessel wall is comparable to the rate to the divertor from parallel transport. The SOL can be thin enough that the recycling neutrals and sputtered impurities from the wall may refuel or contaminate the confinement zone more efficiently than divertor plasma wall interaction. Just inside the SOL is a confinement barrier that produces a sharp pedestal in plasma density and temperature. Understanding neutral transport through the SOL and into the pedestal is key to understanding particle balance and particle and impurity exhaust. The SOL plasma is sufficiently hot and dense to excite and ionize neutrals. Ion and neutral temperatures are high enough that charge exchange between the neutrals and fuel and impurity ions is fast. Excitation of neutrals can be fast enough to lead to nonlinear behavior in charge exchange and ionization processes. In this paper the detailed atomic physics important to the understanding of the neutral transport through the SOL will be discussed.
\end{abstract}

\section{INTRODUCTION}

Atomic and molecular databases have played an important role in the development of magnetic confinement fusion since the inception of research in this area in the 1950's. The importance of basic atomic processes was appreciated early on. Over the decades, magnetic confinement fusion research has progressed to the point where fusion grade plasma conditions are achieved in magnetic confinement devices for short durations. Now the focus of research is on three general areas: 1) further improvement of plasma performance to increase efficiency and economic viability, 2) the achievement of a stationary operating point with fusion grade plasma conditions, and 3 ) the extension of the scientific underpinnings of scaling laws and 
modeling codes to increase confidence in the design of future, burning plasma devices. Atomic physics continues to play a critical role in the diagnostics systems used to measure and understand today's experiments, and is a crucial element of many modeling codes, especially codes used to model the edge plasma. The need for detailed atomic data continues to grow.

At the last ICAMDATA meeting, Dr. Kurt Behringer [1] presented a complete overview of the broad range of atomic and molecular physics that is important for magnetic confinement fusion research. Because fusion grade magnetic confinement plasmas range in temperature from $<1 \mathrm{eV}$ up to $25 \mathrm{keV}$, data is required for low energy processes such as molecular dissociation and high energy processes such as the radiation from highly ionized high $\mathrm{Z}$ elements. Because these plasmas range in density from $<10^{18}$ to $10^{21} \mathrm{~m}^{-3}$, nonlinear and multi-step processes must be considered. The range of atomic species is also very broad. The atomic and molecular physics of deuterium, tritium, and helium, the fuel and ash of the fusion process, is obviously critical. Plasma facing wall materials include low $\mathrm{Z}$ atoms, such as $\mathrm{Li}$ and $\mathrm{Be}$. Graphite, the most commonly used material in today's most advanced research devices, brings with it the need to understand both carbon and hydrocarbon processes. Both molybdenum and tungsten have some advantages over low $\mathrm{Z}$ wall materials and their use is presently being actively being pursued. However contamination of the plasma by these high $\mathrm{Z}$ elements represents a potential loss of energy confinement via strong radiation. Mid $\mathrm{Z}$ elements, e.g. argon, when intentionally injected into fusion grade plasmas have been shown to simultaneously improve total energy confinement while increasing radiative dissipation of energy outflux to the vessel walls. The ionization and radiation physics of these mid and high $\mathrm{Z}$ elements is also needed. All of these atomic data issues have been discussed in Dr. Behringer's paper [1].

In this paper we will emphasize the deep level of detail of atomic data that is needed to accurately model fusion plasmas and interpret spectroscopic diagnostic data. In this brief paper it is not possible to go into the detail important to all the diverse processes listed above, so we will use processes important to the scrape-off layer and edge plasma of a divertor tokamak as an example. This region of the tokamak has received increased attention over the last few years due to two recent discoveries. First, both experimental results and detailed theoretical models of tokamak energy confinement have shown that the achievement of a sharp pedestal in plasma pressure at the edge of the confinement zone significantly reduces thermal transport and improves plasma performance [2]. The physics of this sharp pedestal is not well understood, and is an active area of research. Because of its' proximity to the vessel walls, atomic physics is thought to be an important element to understanding. Second, in the region between the confinement zone and the vessel walls, called the scrape-off layer, enhanced convective transport across the magnetic field has been observed in some operational regimes $[3,4]$. The enhancement can be sufficiently high that the total particle fluence to the main chamber can be comparable to the total particle fluence along the magnetic field to the divertor. The resulting enhanced recycling and impurity sputtering from the wall, combined with the importance of the pedestal in plasma pressure very near the plasma edge, has been a strong motivator for renewed interest in this region. 


\section{THE TOKAMAK SCRAPE-OFF LAYER AND PEDESTAL REGIONS}

The scrape-off layer is a thin layer of plasma separating the hot dense well-confined plasmas from the vessel walls. It must serve two functions, to insulate the vessel walls from the high temperature plasma and to isolate the confinement region from any erosion of material from the walls. All magnetic confinement concepts have a SOL, but in this paper we will focus on the tokamak. The tokamak configuration is the leading magnetic confinement configuration for fusion energy production. It is a toroidal device, with a large, externally imposed toroidal magnetic field and a relatively weaker poloidal magnetic field created mostly from a toroidal current inductively driven in the plasma. The resulting helically shaped magnetic field lines form closed flux surfaces from the center of the current channel outward to near the vacuum vessel walls. A cross section of the vacuum vessel and flux surfaces of a high performance plasma in the DIII-D tokamak [5] is shown in Fig. 1. Toroidally directed coils are placed around the periphery of the vessel to provide vertical stability. In most modern tokamaks these coils are also used to shape the plasma, and to create a null point in the poloidal field. The null point defines a separatrix in the flux surfaces. Inside the separatrix the flux surfaces are closed, and outside of the separatrix they intersect the vessel walls. The plasma inside the separatrix is well confined and is heated to high temperature $(\sim 10 \mathrm{keV})$ using a combination of ohmic heating due to the plasma current and auxiliary heating from injected energetic neutral beams or rf power. The plasma confinement is not perfect, and heat and particles leak from the confined region, across the separatrix, and ultimately to the vessel walls. The region of plasma near the point where the open field lines intersect the wall is known as the divertor, while the region of plasma lying on the field lines just outside of confinement zone is known as the scrape-off layer (SOL). In the conventional picture of the SOL and divertor, plasma flows along the field lines much faster than across the field lines. The heat and particles lost across the separatrix are directed to the divertor plates where they recycle back into the plasma as neutrals and sputter divertor plate material into the plasma. One of the reasons behind the divertor design is the large spatial separation between the separatrix and the divertor strike plates that can be achieved. The divertor is obviously a place rich in atomic and molecular physics and has been the focus of much research for many years [6].

The SOL is a region of lower plasma density than either the core plasma or the divertor plasma, being around $10^{18}$ to $10^{19} \mathrm{~m}^{-3}$, with electron and ion temperatures not well equilibrated and lying typically between 10 and $200 \mathrm{eV}$. Profiles of plasma electron density and temperature and ion temperature in the region of the edge pedestal and SOL are shown in Fig. 2 for a typical high-confinement mode (H-mode) plasma in DIII-D. In an H-mode plasma, a region of very low transport is established just inside the separatrix. This narrow region of low transport appears spontaneously and is related to the appearance of a strong radial electric field. It results in the development of a sharp pedestal in plasma density and pressure. Near the separatrix the plasma density and temperature are seen to decrease exponentially away from the core plasma, with decay lengths between $0.5 \mathrm{~cm}$ to a few $\mathrm{cm}$. Since the distance to the nearest vessel wall is usually several $\mathrm{cm}$, wall fluxes were thought to be low. In 


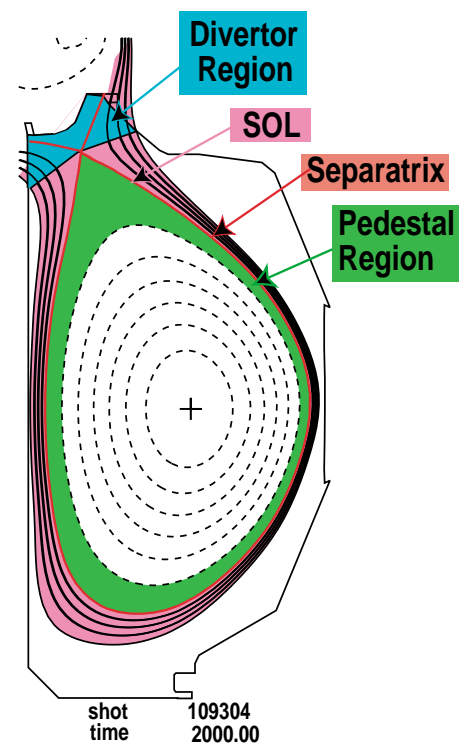

FIGURE 1. A cross section of the DIII-D vessel is shown along with reconstructed flux surfaces of an upper single null diverted discharge. The separatrix, divertor, SOL, and pedestal regions are identified.

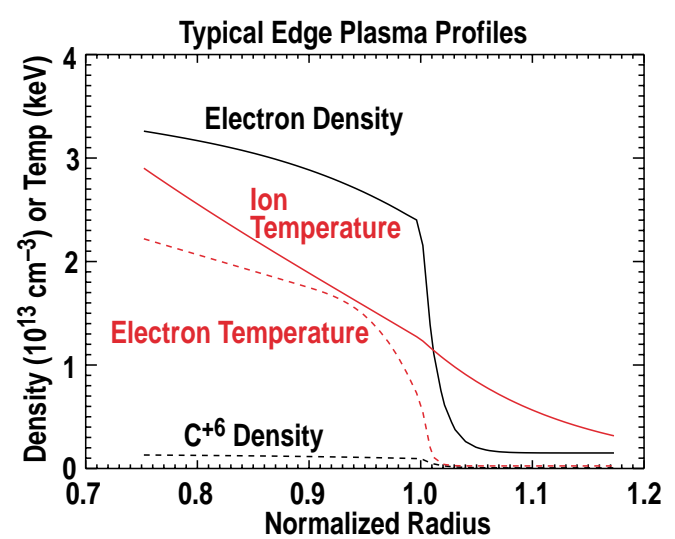

FIGURE 2. The radial profiles of plasma density, electron and ion temperatures are shown as a function of normalized radius. The value $\rho=1$ is at the separatrix.

addition, ionization path lengths of slow neutrals ( $1 \mathrm{eV}$ or less) are short and so the core plasma is protected from wall sources. Recent data on SOL phenomena from the Alcator C-Mod [3] tokamak at higher than usual plasma density clearly showed that beyond the separatrix, the density and temperature scale lengths could become very long. In such cases the plasma flux across the SOL to the vessel wall was very high, completely dominating the flux to the divertor. Studies on DIII-D indicate that crossfield transport in the far regions of the SOL can result in wall flux being competitive with divertor flux at more moderate plasma density [4]. The existing data is sufficient to show that the main chamber can be a significant source of refueling and contamination, even in a diverted tokamak. Work continues to define both the regimes in which this fast cross-field transport is important, and the mechanisms responsible.

The separatrix lies at the base of a sharp edge pedestal. Most apparent in H-mode plasmas, the pedestal occupies only a few centimeters of distance, but within this narrow zone the electron density and temperature rise sharply from typical separatrix values of 1 to $2 \times 10^{19} \mathrm{~m}^{-3}$ and $50-100 \mathrm{eV}$ up to several $10^{19} \mathrm{~m}^{-3}$ and over $1 \mathrm{keV}$ at the top of the pedestal. This pedestal has been seen to play important roles in enhancing energy confinement and improving stability at high plasma pressure. The pedestal arises due to a zone of reduced transport near the edge [7], however the physics that determines the pedestal height and width is not well understood. Because neutral deuterium penetrates into the pedestal, it is hypothesized that atomic physics may play a role in determining the pedestal parameters. 


\section{ATOMIC PHYSICS IMPORTANT TO NEUTRAL RECYCLING FROM THE MAIN CHAMBER WALL}

The sources of atomic and molecular neutrals important for the SOL include recycling of the primary ion species at the main chamber wall, chemical and physical sputtering of the plasma facing surfaces, gas puffing of the primary species, intentionally puffed impurity species, and leakage of neutrals from the divertor region. In the plasma, the neutrals undergo many collisional processes with plasma electron, ions, and impurity ions. One of the more interesting transport problems is that of hydrogen, the primary fueling species. (We will not distinguish between hydrogen isotopes in this discussion.) The neutral hydrogen recycles from the plasma-facing wall as molecular $\mathrm{H}_{2}$ and as atomic $\mathrm{H}$. The recycling molecules result from surface recombination of adhered $\mathrm{H}$ and leave the wall at thermal energies. The recycling $\mathrm{H}$ results mostly from reflection of incident ions and will have higher initial kinetic energy. At thermal kinetic energy, the molecules have a short mean free path for electron impact dissociation, resulting in atomic $\mathrm{H}$ at Franck-Condon energies determined by the dissociation process. It is the transport of the atomic $\mathrm{H}$ that dominantly determines the fueling (re-ionization) profile and the flux of energetic charge exchange neutrals back to the wall.

At first glance, the collisional processes of atomic $\mathrm{H}$ with the plasma seem fairly simple. As the atoms drift away from the wall, they can be ionized by electron and proton impact, they can be ionized by impact and charge exchange with impurity ions, and they exchange velocity with the plasma ions through the symmetric charge exchange process. At the collision energies typical for the SOL, ion impact ionization is negligible, so only the electron and impurity charge exchange collisions contribute to the loss of atomic $\mathrm{H}$ and the net refueling of the plasma. Since the plasma ion temperature typically exceeds the energy of the neutrals, the symmetric charge exchange process serves to change the velocity of the neutrals and increase their average energy. This process increases the depth of penetration of neutrals and results in a flux of energetic neutrals back to the main chamber wall.

The atomic physics is significantly enriched due to the fast electron excitation rates for atomic $\mathrm{H}$ near the separatrix and in the pedestal region [8]. In the SOL, the excitation process is sufficiently fast to produce an average excitation fraction of about $10^{-3}$ in the first several principal quantum levels of the neutral $\mathrm{H}$ [9]. Because ionization rates of atomic $\mathrm{H}$ are a very strong function of excitation level, even this rather small excitation fraction will lead to a significant increase in the total ionization rate. The fractional populations of the $n=2$ and $n=4$ levels of atomic $\mathrm{H}$ as a function of electron density at electron temperatures of $10,100 \mathrm{eV}$, and $1000 \mathrm{eV}$ as obtained from the ADAS data base are shown in Fig. 3. The charge exchange cross section and electron impact ionization cross sections for $n=2-5$ are shown as a function of electron temperature in Fig. 4. These data are obtained from scaling relations found in the data series entitled "Atomic and Plasma-Material Interaction Data for Fusion", Volume 4, published by the IAEA. The cross sections increase dramatically with $\mathrm{n}$. The charge exchange cross sections increase very dramatically, scaling as $\mathrm{n}^{4}$ at collision energies relevant to the SOL and pedestal region. 

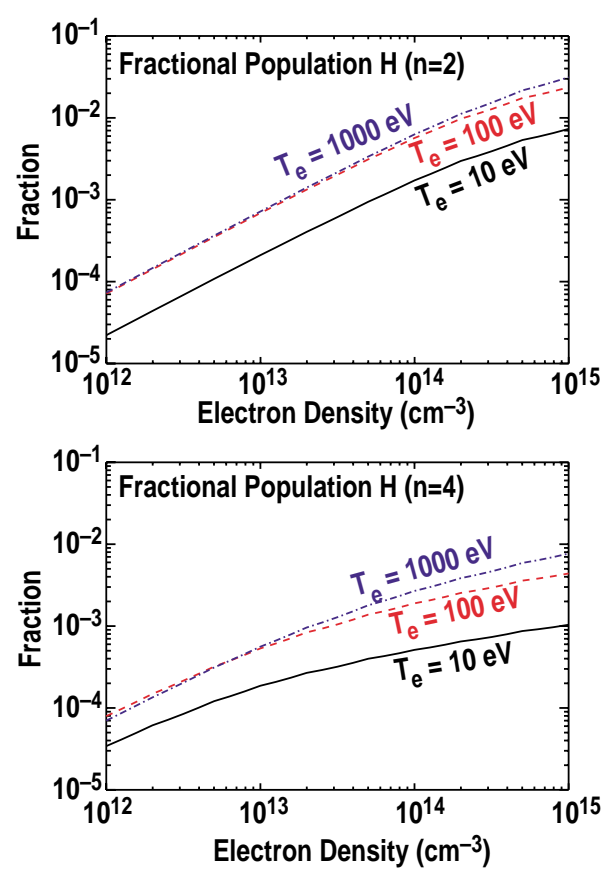

FIGURE 3. Excitation fraction of the $n=2$ and $\mathrm{n}=4$ levels of atomic $\mathrm{H}$ as a function of electron density at an electron temperature of $100 \mathrm{eV}$.
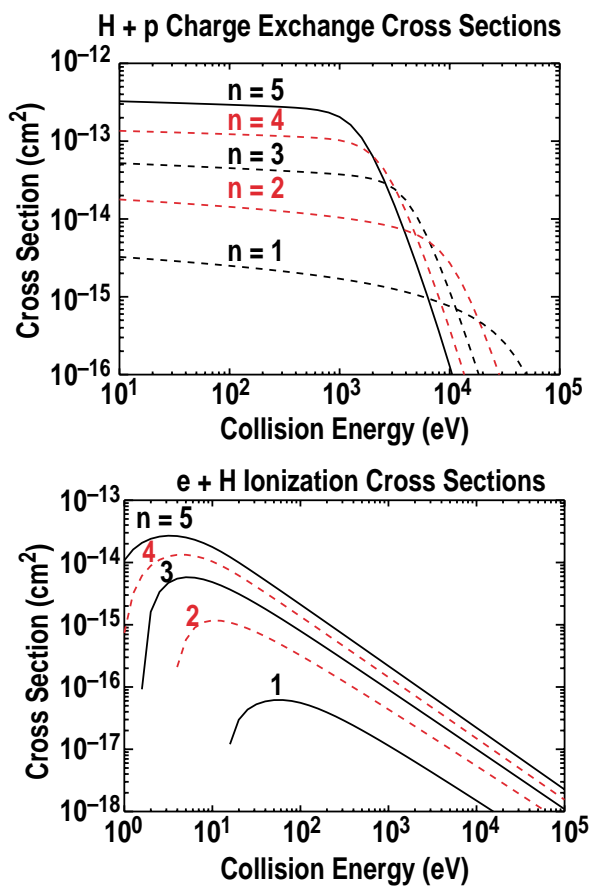

FIGURE 4. The electron impact ionization and proton charge exchange cross sections for collisions with excited atomic $H(n=1-5)$ is shown as a function of collision energy.

The total electron ionization and charge exchange (proton and carbon impurity) rates including the excited state contributions are shown in Fig. 5 as a function of plasma density. In the rather modest densities typical of the tokamak pedestal region, the inclusion of the excited state contribution is seen to double the total charge exchange rate. In this region, the proton charge exchange rate becomes comparable to, and in some cases greater than, the electron impact ionization rate. The ionization rate
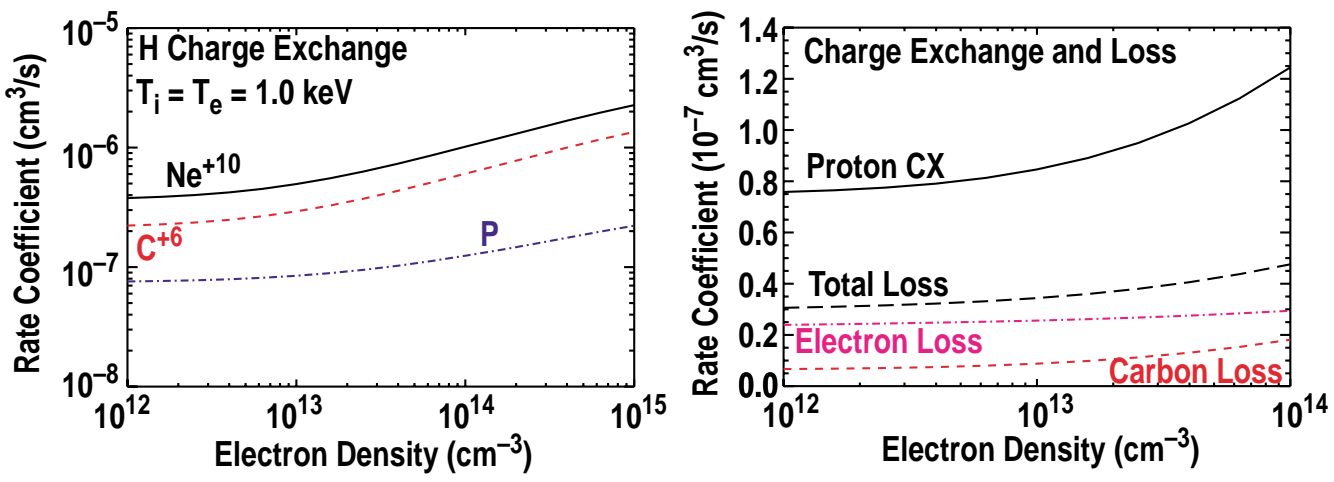

FIGURE 5. The rate coefficients for electron impact ionization and total charge exchange for protons and $\mathrm{C}^{+6}$ with atomic $\mathrm{H}$ including the effect of excited states up to $\mathrm{n}=5$ are shown as a function of plasma density at an electron temperature of $1.0 \mathrm{keV}$. The scaling relations found in the IAEA data series are used, along with excitation fractions from the ADAS database 
due to charge exchange with carbon impurity ions is also large in this region. Even though the carbon concentration is typically around $3 \%$, it can contribute over $50 \%$ to the total loss rate of atomic $\mathrm{H}$.

The accuracy of the data should also be of some concern. As an example of the typical level of accuracy, the charge exchange cross section of atomic $\mathrm{H}$ with fully stripped neon is shown as a function of energy in Fig. 6. Neon was first used in magnetic fusion to enhance the radiation in the core plasma and reduce the heat flux across the separatrix and to the plasma facing surfaces. During these enhanced radiation experiments, it was noticed that impurity injection also improved the energy confinement and overall plasma performance [10]. Cross sections obtained from the scaling relation are compared to classical trajectory Monte Carlo calculations [11]. The scaling relations are derived from a critical evaluation of available theory and experimental data. The charge exchange cross sections for the ground state obtained using the scaling relations are found to be somewhat higher than those from the CTMC calculations in the energy range of interest to the SOL.

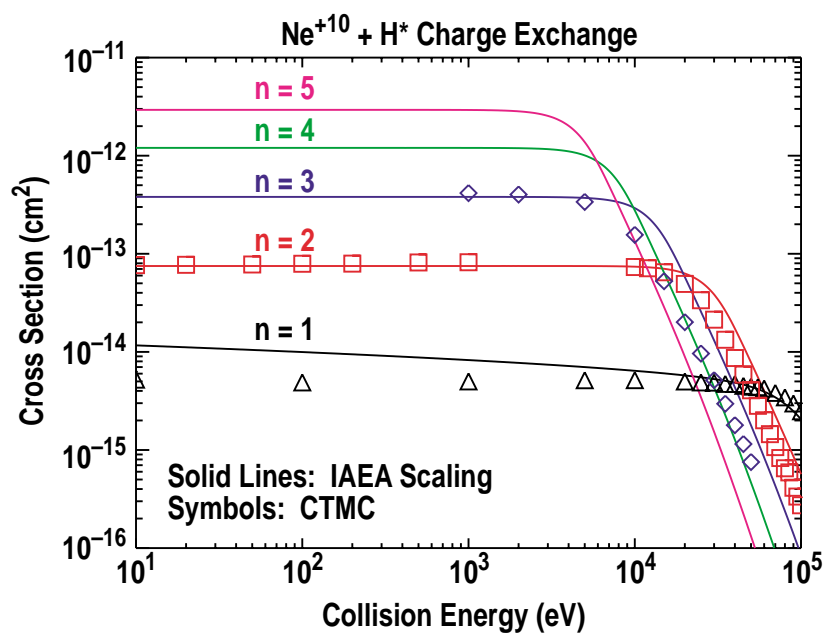

FIGURE 6. The charge exchange cross section for collisions of atomic $\mathrm{H}(\mathrm{n}=1-5)$ with $\mathrm{Ne}^{+10}$ are shown as a function of collision energy from the scaling relations in the IAEA data series and from CTMC theoretical calculations.

\section{DISCUSSION}

Using the relatively simple example of the transport of atomic neutrals through the SOL and edge plasma, we find that much more atomic data than just the ionization and charge exchange cross sections for the ground state is needed. Because the plasma density and temperature are sufficient to provide excited state population fractions $\sim 10^{-3}$, and because charge exchange cross sections scale as $n^{4}$, we need these cross sections and their energy dependence for excited states of the neutral up to at least $\mathrm{n}=5$.

Interpretation of spectroscopic data from impurities in the edge and core plasma requires another level of detail. Impurity line emission in the visible region of the spectrum is frequently used to monitor impurity ion species in the edge plasma. Usually these lines result from transitions from highly excited states. Charge exchange between impurity ions and excited atomic $\mathrm{H}$ may be a significant contributor to the 
production of such line emission, so state resolved charge exchange cross sections are needed to accurately model the spectroscopic data. Visible emission of helium-like carbon ions in the divertor of DIII-D resulting from charge exchange with excited states of atomic $\mathrm{H}$ has been used to measure the parallel flow velocity of carbon between the divertor and SOL [12]. Also, excited states of atomic $\mathrm{H}$ in energetic neutral beams used for charge exchange spectroscopy have been shown to make a significant contribution to the photon production cross section in collisions of $\mathrm{Ne}^{+10}$ [13]. An example of product state resolved cross sections for $\mathrm{H}(\mathrm{n}=2)$ collisions with $\mathrm{Ne}^{+10}$ calculated using the CTMC model is shown in Fig. 7.

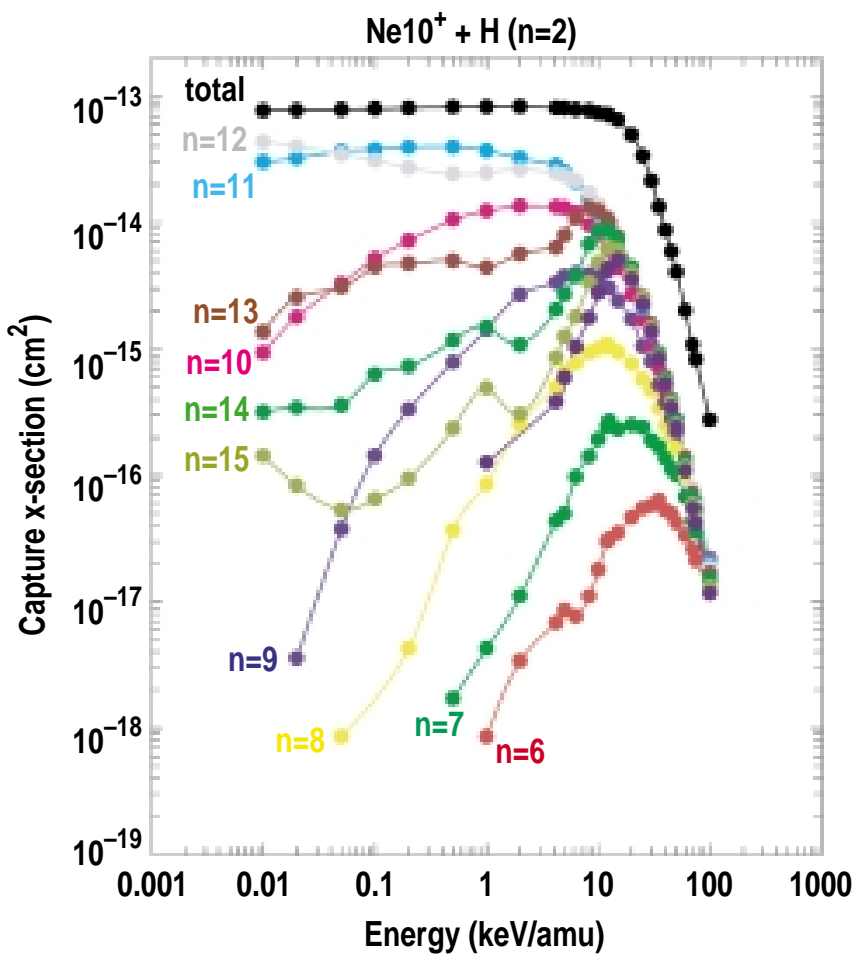

FIGURE 7. The product state resolved charge exchange cross sections for $\mathrm{H}(\mathrm{n}=2)+\mathrm{Ne}^{+10}$ from CTMC calculations are shown as a function of collision energy.

The need for such detailed charge exchange data to accurately model fusion plasmas and to interpret spectroscopic diagnostics has been understood for some time. Because magnetic confinement fusion plasmas cover a broad range of plasma density and temperature, such detail is needed in all the processes occurring in the plasma. The atomic physics community has been very responsive to the needs of plasma modelers for many years, and the databases, such as the ADAS database and the IAEA data center, continue to grow in response to the needs of the fusion community and to the resources provided by the atomic physicists.

In a similar fashion, a deep level of detail is needed to understand molecular processes in the edge plasma. Such processes are important for recycling, recombination, chemical sputtering, co-deposition and tritium entrapment. 


\section{ACKNOWLEDGMENTS}

This is a report of work supported by the U.S. Department of Energy under Contract DE-AC03-99ER54463.

\section{REFERENCES}

1. Behringer, K., "Atomic Physics Requirements in Fusion Research," Atomic and Molecular Data and Their Applications, edited by K.A. Berrington and K.L. Bell, CP543, American Institute of Physics 1-56396-9718/00, Melville, New York, 2002, p. 129.

2. Pedestal reference.

3. LaBombard, B., Umanski, M. V., Boivin, R. L., et al., Nucl. Fusion 40, 2041 (2000).

4. Boedo, J. A., Rudakov, D. L., Moyer, R. A., Krasheninnikov, S., Whyte, D. G., McKee, G. R., et al., Phys. Plasmas 8, 4826 (2001).

5. Luxon, J. L., and Davis, L. G., Fusion Technol. 8, Part 2A, 441 (1985).

6. Stangeby, P. C., The Plasma Boundary of Magnetic Fusion Devices, edited by P. Stott and H. Wilhelmsson, Institute of Physics Publishing, Bristol, United Kingdom and Philadelphia, Pennsylvania, 2000.

7. Wagner, F., et al., Phys. Rev. Lett. 49,1408 (1982).

8. Johnson, L. C., Astrophys. J. 174 (1972).

9. Summers, H. P., ADAS, Atomic Data and Analysis Structure, Originally developed by the Jet Joint Undertaking, $1^{\text {st }}$ Edition User Manual, 1994.

10. Ongena, J., Messiaen, A. M., Unterberg, B., et al., Plasma Phys. and Control. Fusion 41, A397 (1999).

11. Olson, R. E., J. Phys. B 13, 483 (1980).

12. Zaniol, B., Isler, R. C., Brooks, N. H., West, W. P., "Comparison of Experimental Measurement of Carbon Flow Velocities and Temperatures to UEDGE Predictions in the DIII-D Divertor," Bull. Am. Phys. Soc. 46, 148 (2001).

13. Whyte, D. G., Wade, M. R., Finkenthal, D. K., et al., Nucl. Fusion 38, 387 (1998). 